\title{
UN ESTUDIO DE LA ABUNDANCIA DEL RONSOCO (H ydrochaeris hydrochaeris) EN LA RESERVA NACIONAL PACAYA - SAMIRIA Y PROPUESTA DE UN METODO DE CENSO
}

\author{
Pekka Soini ${ }^{*}$
}

\section{RESUMEN}

Estimados de la abundancia del ronsoco (Hydrochaeris hydrochaeris) en la Reserva N acional Pacaya-Samiria, durante varios años, indican que para el curso medio del río Pacaya la abundancia general es de 3.2 animales $/ \mathrm{km}$ río, con una densidad ecológica, en su hábitat óptimo, de 25.8 animales $/ \mathrm{km}^{2}$ (8.3 animales $/ \mathrm{km}$ río). El número de hembras reproductivas fue igual al de grupos y parejas, siendo la abundancia general de 0.6 hembras $/ \mathrm{km}$ río y la densidad ecológica de 3.1 hembras $/ \mathrm{km}^{2}$. Las márgenes de tipishcas y cochas sostienen poblaciones muy bajas, constituyendo el habitad menos favorable para la especie. La creciente excepcionalmente grande del río en el año 1986 y la epidemia de tripanosomiasis que le siguió, redujeron la población del hábitat óptimo a 4.1 animales $/ \mathrm{km}^{2}$, se propone un método de censo para las poblaciones amazónicas del ronsoco.

\section{ABSTRACT}

The population density of capybara (Hydrochaeris hydrochaeris) was investigadet over several years on the Pacay river, in the Pacaya-Samiria N ational Reserve, Perú. The general density for the middle Pacaya was estimated at 3.2 animal $\mathrm{s} / \mathrm{km}^{2}$ ( 8.3 animal $\mathrm{s} / \mathrm{km}$ of river) in the optimum habitat. The number of reproductive females was equal to that of groups and pairs, with a general density of 0.6 females $/ \mathrm{km}$ of river and an ecological density of $3.1 \mathrm{femal} \mathrm{es} / \mathrm{km}^{2}$. The oxbow lake margins constitute a less favorable habitad for the capybara, and support only very low population densities. The exeptionally high flood of 1986, and a trypanosomiasis epidemy that ensued, reduced the population density in the optimum habitat down to 4.1 animals $/ \mathrm{km}^{2}$. A census method suited for the A mazonia capybara populations is proposed.

\footnotetext{
Investigador -IIAP
} 
Palabras Claves: Hydrochaeris hydrochaeris, censo, abundancia, densidad, población, Reserva Nacional Pacaya-Samiria.

\section{INTRODUCCION}

Debido a la importancia económica real o potencial del ronsoco Hydrochaeris hydrochaeris, varios investigadores han realizado estudios de la abundancia y densidad poblacional de la especie en los Llanos de Venezuela (Ojasti, 1973; Eisenberg et al, 1979; Macdonald, 1981; Cordero y Ojasti, 1981), Colombia (Jorgenson, 1986) y en el Pantal de Mato Grosso, Brasil (Shaller y Crawshaw, 1981; Schaller, 1983).

Sin embargo no se ha tenido, hasta ahora, información cuantitativa referente a su abundancia en el ecosistema de bosque lluvioso amazónico.

En los llanos y en el pantanal el ronsoco habita mayormente lugares abiertos, donde los animales pueden ser avistados y contados a plena luz del día, avanzando a pie, caballo o vehiculo motorizado por los caminos 0 a través de la Ilanura (Ojasti, 1973; Eisenberg et al., 1979; Cordero y Ojasti, 1981; Macdonald, 1981; Jorgenson, 1986; Schaller, 1983; Schaller y Crawhasw, 1981).

En la A mazonía, el ronsoco habita las márgenes bajas e inundables de los ríos y otros ambientes acuáticos. En contraste con las poblaciones de las Ilanuras abiertas, los individuos permanecen, durante el día, dentro de la espesura de la vegetación ribereña.

En estos ambientes la vegetación es enmarañada, dificultando la movilización, y la visibilidad se reduce a pocos metros; por lo que la ejecución de censos poblacionales de la especie requiere una metodología diferente de la que se aplica en otros ecosistemas.

Entre 1979 y 1991 se realizaron censos poblacionales y estudios sobre la ecología y dinámica poblacional del ronsoco en el río Pacaya, Reserva Nacional PacayaSamiria (Soini y Soini, 1992; Soini, 1993).

Se presenta, en éste trabajo, los resultados referentes a la abundancia de la especie y se describe un método sencillo de censo y que requiere relativamente poco esfuerzo. 


\section{METODOS}

El estudio fue realizado principalmente en el área de la Estación Biológica de Cahuana, ubicada en el curso medio del río Pacaya, abarcando un tramo de 3 $\mathrm{km}$ del río y los terrenos colindantes, denominado área $\mathbf{A}$; y secundariamente en un tramo contiguo del río, de $12 \mathrm{~km}$ de longitud, denominado área B (figura 1).

El área de estudio, ubicado en la planicie inundable, corresponde a la formación "bosque ripícola" o "tahuampa de agua negra" (Encarnación 1985), sujeto a inundación estacional desde enero hasta junio. Una descripción del área y del hábitat del ronsoco fue dada por Soini y Soini (1992).

LoS censos poblacionales se realizaron en los años 1979, 1980, 1988, 1990 y 1991 para el área A y en 1984 para el área B, durante la temporada de estiaje del río y hasta la temporada inicial de la creciente (julio a diciembre), procurando ubicar todos los animales.

Para los fines del censo, se realizaron semidiariamente recorridos diurnos y nocturnos, a pie y canoa, por los diferentes sectores del área de estudio, registrando, en un mapa de escala grande $(1: 5,000)$, las ubicaciones y desplazamientos (incluyendo de huellas frescas) y composición de todas las unidades sociales de ronsoco encontradas.

Los registros acumulados permitieron luego reconocer individualmente cada uno de los grupos presentes en el área; el reconocimiento individual de los animales solitarios, sin embargo, no siempre fue posible.

\section{RESULTADOS Y DISCUCION}

\subsection{Densidad poblacional}

Los censos intensivos de la población del área $A$ indicaron que en setiembre de 1979 y 1980 la población consístia en 23 y 27 animales, respectivamente (Cuadro 1), con una abundancia promedio de 8.3 animales $/ \mathrm{km}$ río. 
La extensión total del hábitat adecuado para el ronsoco dentro del área $\mathrm{A}$, incluyendo el espejo de agua, comprende aproximadamente 97 ha (figura 1); lo que da una densidad ecológica de 25.8 animales $/ \mathrm{km}^{2}$.

Debido a una al ta mortalidad entre las crías (Soini, 1993) las camadas sufren rápida reducción inicial de tamaño o tienen, muchas veces, solo una existencia efímera en la población, ocasionando una fluctuación concomitante muy acentuada en el tamaño de la población local, lo que puede hacer más difícil una comparación entre poblaciones y/o tiempos.

Esto puede obviarse excluyendo, para fines de comparación, el componente infantil de la población. Para el área $A$, los valores correspondientes serían de 5.0 animales no infantes/ $\mathrm{km}$ río y 15.5 animales no infantes/ $\mathrm{km}^{2}$.

Otro parámetro de interés para el estudio y manejo de las poblaciones es la proporción o densidad de hembras reproductivas.

En este censo, un grupo o pareja fue considerada reproductiva si contenía uno o más infantes o juveniles en algún momento dentro del período de censo (julio - diciembre).

En contraste con lo observado en los Llanos (Ojasti, 1973; Azcárate, 1980; M acdonald, 1981; Jorgenson 1986) y en el pantanal (Schaller y Crawshaw, 1981), se encontró que en el río Pacaya sólo una hembra criaba en cada grupo (Soini, 1993), por lo que el número de hembras reproductivas en la población es igual al número de grupos y parejas reproductivos.

En el área A hubo dos grupos reproductivos en 1979 y 4 en 1980, siendo el promedio respectivo 3.0 , o sea 1.0 grupos $/ \mathrm{km}$ río. La densidad ecológica correspondiente fue de 3.1 grupos (o hembras reproductivas) $/ \mathrm{km}^{2}$.

El censo realizado en el área B reportó, en setiembre de 1984, una población total de 39 ronsocos (cuadro 2), siendo la abundancia correspondiente 3.2 animales $\mathrm{km} / \mathrm{rí}$. La falta de información precisa de la extensión total de hábitat adecuado para el ronsoco en ese tramo, no nos permite estimar la densidad ecológica para el área B.

Excluyendo el componente infantil de la población, la abundancia para el área B sería de 2.3 animales $\mathrm{km} /$ río. El número total de grupos y parejas reproductivas fue de 7,10 que equivale a 0.6 grupos 0 hembras reproductivas/km río. 
L os resultados de éstos censos indican que la abundancia de ronsocos fue más del 100\% mayor en el área A que lo encontrado en el área B en 1984. A unque no se tienen datos cuantitativos precisos al respecto, las observaciones anuales en el área A indican que en 1984 la abundancia fue parecida a lo encontrado en 1979 - 1980.

Al parecer, la diferencia entre las dos áreas se debe principalmente a que casi toda el área A cuenta con amplios gramalotales, con predominancia de la graminea Echinochloa polystachya, el alimento principal del ronsoco (Soini y Soini 1992), asociado con orillas bajas y abiertas, que se forman en los sectores meandrosos del curso del río, constituyendo el habitat óptimo del ronsoco.

El área B incluye tramos menos favorables, donde las orillas abiertas están ausentes 0 son muy reducidas y por lo tanto tienen menor capacidad de carga de ronsocos. En vista de que el área $\mathrm{B}$ presenta mejor el medioambiente del Pacaya medio, concluimos que la abundancia encontrada en éste área refleja mejor la abundancia general de la especie; la densidad poblacional encontrada en el área A reflejaría más bien la abundancia que alcanza en los lugares de hábitat óptimo.

Observaciones anuales realizadas en la cocha o tipishca de Cahuana, un meandro abandonado del rió de $12 \mathrm{~km}$ de longitud, revelaron que en sus márgenes vivían muy pocos ronsocos: al gunos animales solitarios y solamente una pareja reproductiva con su prole, ocupando el único gramalotal existente (figura 1). La escasez se debía evidentemente a la ausencia de gramalotales de Echinochloa polystachya.

L a formación y sostenimiento de éstos gramalotes están ligados al proceso de deposición activa de sedimentos fluviales, de las aguas blancas 0 aguas de mezcla, sobre las orillas (obs. pers.). En las cochas y tipishcas que no tienen influencia de las aguas cargadas de sedimento, los gramalotales están generalmente ausentes. Esto nos permite concluir que por regla general el ronsoco es mucho menos abundante en las márgenes de las tipishcas y cochas que en las del curso del río.

L a creciente excepcionalmente grande del río Pacaya en 1986, y una epidemia subsiguiente de tripanosomiasis, causaron una drástica reducción de la población de ronsocos (Soini 1993). 
En 1988, la población del área $A$ se había reducido a $8 \pm 2$ animales; y al finalizar el estudio, en 1991, sólo 4 animales vivían en el área; indicando que la población no mostraba aún señales de recuperación. Observaciones circunstanciales en los años subsiguientes indicaron un leve incremento de la población.

Los resultados demuestran que el tamaño de una población puede mostrar una fluctuación muy acentuada, debido a la poca estabilidad del hábitat que ocupa el ronsoco: en una década de observación en el área $A$, la densidad fluctuaba entre 4.1 y 28.5 animales $/ \mathrm{km}^{2}$.

\subsection{Comparación con otras poblaciones}

No existen datos comparativos de otras localidades dentro del ecosistema del Bosque Lluvioso, a excepción de Janson y Emmons (1990), quienes reportaron una densidad de 1.6 ronsocos $/ \mathrm{km}^{2}$ para Cocha Cashu, Parque $\mathrm{N}$ acional del $\mathrm{M}$ anu, basado en un censo general de mamíferos.

A unque aparentemente su estimado no se deriva de un estudio o censo poblacional intensivo, nos sugiere que el ronsoco es más abundante en la Reserva Nacional Pacaya-Samiria que en el Manu.

Los censos poblacionales realizados en los Llanos de $V$ enezuela reportan densidades generales que varían de 10 a 60 animales/km2 (Eisenberg et al., 1979; M acdonald, 1981; Ojasti, 1983).

Durante la estación seca las poblaciones se concentran alrededor de los cuerpos permanentes de agua y las densidades ecológicas varían mayormente entre 100 y 200 animales/km2, alcanzando excepcionalmente hasta 360 animales/km2 (Ojasti, 1973, 1983; Cordero y Ojasti, 1981). Es evidente que el ecosistema de los Ilanos sostiene poblaciones más grandes de ronsocos que el bosque lluvioso; indudablemente debido a la mayor disponibilidad de hábitat apropiado.

En el Pantanal de Mato Grosso, Schaller y Crawshaw (1981) hallaron una densidad general de 12.5 ronsocos $/ \mathrm{km}^{2}$. En otra localidad, dentro del mismo ecosistema, Schaller (1983) encontró una población de 30-35 ronsocos en un tramo de $8 \mathrm{~km}$ de orilla de pantanal inundado, lo que equivaldría a 3.7-4.4 animales/km. A unque el hábitat es muy similar al de los Ilanos, las densidades 
poblacionales encontradas son relativamente bajas, semejándose más a las densidades observadas en el río Pacaya.

Esto podría deberse a que ambas poblaciones censadas en el pantanal habían sido afectadas por epidemias de tripanosomiasis (Schaller y Crawshaw, 1981; Schaller 1983).

\section{DESARROLLO Y PROPUESTA DE UN METODO DE CENSO}

Las observaciones del comportamiento del ronsoco en el Pacaya demostraron que en la temporada anual de estiaje del río (agosto-octubre) toda la población frecuenta asiduamente las orillas bajas y descubiertas del río para pacer, bañarse y jugar.

Esto lo hacen mayormente de noche, aproximadamente a partir de las 18:30 horas, pero también con cierta frecuencia en las primeras horas de la mañana. Cuando de noche se les ilumina con una linterna desde el río, generalmente quedan inmóviles sobre la orilla, por lo menos inicialmente, lo que a menudo permite contar bien los individuos y clasificarlos en adultos y juveniles. Basándonos en estas observaciones, desarrollamos un método de censo nocturno que consiste en lo siguiente:

Dos censadores, equipados con linternas, recorren paralelamente, en canoas separadas, un tramo predeterminado de río, de tres o más $\mathrm{km}$ de longitud, a favor de la corriente, cada uno manteniéndose a una distancia aproximada de $10 \mathrm{~m}$ de su orilla respectiva. Mientras avanzan lenta y silenciosamente, revisan visual mente la orilla iluminándola intermitentemente con la linterna. A I encontrar ronsocos, toman nota de la ubicación y del número total de animales presentes, haciendo una diferenciación entre adul tos y juveniles.

El recorrido debe ser repetido varias veces, pero una sola vez por noche, o una vez en las primeras horas de la noche y otro en las últimas, para que la concurrencia de los ronsocos a la orilla en las horas del recorrido no sea afectada por el recorrido anterior.

En setiembre - octubre de 1979 realizamos 11 recorridos nocturnos de censo por el tramo A del río. El cuadro 3 muestra los resultados de los recuentos respectivos y el cuadro 1 muestra la composición y tamaño real de la población censado. Como se puede ver en el cuadro 3, el máximo recuento obtenido en un 
recorrido fue de 16 animales, lo que representa el $70 \%$ de la población total existente en ese tramo.

El examen de los cuadros revela que en ninguno de los 11 recorridos se hallaron simultáneamente presentes, en la orilla, los dos grupos familiares que vivían en el área, como tampoco las dos parejas; mientras que los tres solitarios estaban presentes en tres oportunidades.

Al parecer, esto no fue debido a mutuo evitamiento, ya que las áreas de ocupación de las dos familias y parejas, respectivamente, no se sobreponían; más bien parece haber sido fortuito, debido a un número insuficiente de recorridos de censo.

Con un número adecuado de recuentos (más de 20 por tramo de censo), realizados en la temporada de máximo estiaje y en la forma que acabamos de describir, se obtendrá un censo completo o casi completo de la población en el tramo muestreado.

Para verificar si la máxima cuenta obtenida en un recorrido incluye todas las unidades sociales encontradas por lo menos una vez en el transcurso del periodo de censo, y para hacer cualquier ajuste necesario al estimar el tamaño de la población, o ampliar la muestra mediante recuentos adicionales, es necesario realizar un procedimiento complementario a los recuentos nocturnos, de la siguiente manera:

1. Dibujar en un mapa o croquis del tramo de censo las ubicaciones de todos los encuentros con ronsocos;

2. Identificar, en lo posible, individualmente los grupos encontrados por su composición y características de comportamiento (grupo arisco, manso, etc.) D etalles sobre características físicas resaltantes de los individuos, tal es como sexo, tamaño relativo, aspecto externo, cicatrices grandes, estado de salud, etc., pueden ser muy útiles para la identificación de individuos y grupos; por ejemplo, a los machos adultos se les reconoce por la presencia de una glándula cebácea, conspicua, sobre el hocico.

Información adicional sobre la composición de un grupo y tamaño (edad) de sus integrantes juveniles, puede obtenerse examinando (de día) el tamaño de las huellas de las pisadas que los integrantes del grupo dejaron en la orilla. 
En el cuadro 4 damos la relación aproximada del tamaño de las huellas del pie trasero y la edad del animal, basada en el seguimiento del desarrollo de las crías de algunos grupos silvestres en el área $\mathrm{A}$ y el crecimiento del pie de una cría criada a mano en la estación biológica de Cahuana.

Los ronsocos se encuentran también, con cierta frecuencia, en las orillas expuestas, en las primeras horas de la mañana; por lo que realizamos 17 recorridos matutinos de censo en agosto - octubre de 1983 y 29 recorridos en agosto - octubre de 1984 en el tramo B, de $12 \mathrm{~km}$ de longitud (Figura 1).

L a máxima cuenta obtenida en 1983 fue de diez animales (cinco adultos y cinco juveniles) y en 1984, 12 ( siete adultos y cinco juveniles).

Esta última cifra representa solo el $31 \%$ de la población que, según el estudio más intensivo (cuadro 2), habitaba en ese tramo del río. Esto demuestra la ineficiencia de éste método en comparación con el método de recuentos nocturnos.

U na vez determinado el número total de animales en un tramo de censo, se puede obtener un índice de abundancia, que permite comparar la abundancia de ronsocos entre diferentes lugares y/o tiempos, expresado en número de animales por $\mathrm{km}$ de cauce del río (u otro cuerpo de agua), dividiendo el total de animales por la longitud del tramo de censo. 


\section{BIBILIOGRAFIA}

AZCARATE, T. 1980. Sociobiología y manejo de capybara (Hydrochoerus hydrochaeris) Doñana Acta Vertebrata, 7: pp. 13 - 28.

CORDERO, G.A; OJASTI, J. 1981. Comparison of capybara populations of open an forested habitats, J. Wildl. M anag., 45: pp. 267 - 271.

EINSENBERG, J.F.; O'CONNELL, M. A.; AUGUST, P. V. 1979. Density, productivity and distribution of mammals in two Venezuelan habitats. pp. 187 - 207. en J.F. Einsenberg (ed), Vertebrate Ecology in the Northern Neotropics. Smithsonian Press, Washington.

ENCARNACION, F. 1985. Introducción a la flora y vegetación de la A mazonía Peruana: estado actual de los estudios, medio natural y ensayo de una clave de determinación de las formaciones vegetales en la llanura amazónica. Candollea, 40: pp. 237 - 252.

JANSON, C.H. Y EM MOMS, L.H. 1990. Ecological structure of the nonflying mammal community at Cocha $\mathrm{C}$ ashu Biological Station, $\mathrm{M}$ anu $\mathrm{N}$ ational Park, Perú. pp. 314 - 338 en A. Gentry (ed). Four Neotropical Rainforests. Y ale Univ. Press. N ew Haven.

JORGENSON, J. P. 1986. Notes on the ecology and behavior of capybaras in northeastern Colombia. Vida Silv. Neotrop., 1: pp. 31 - 40.

MACDONALD. D.W. 1981. Dwindling resources and the social behaviour of capybaras (Hydrochoerus hydrochaeris) (M ammalia). J. Zool. (London), 194: pp. $371-391$.

OJASTI, J. 1973. Estudio biológico del chiguire o capibara. 276 pp. Fondo $\mathrm{N}$ acional de Investigaciones A gropecuarias, Caracas.

OJASTI, J. 1983. Ungulates and large rodents of South A merica. pp. 427 - 439. En F. Bourleire (ed). Tropical savannas. Elsevier, A msterdam.

SCHALLER, G.B. 1983. Mammals and their biomass on a Brazilian ranch. Arq. Zool. (Sao Paulo), 31: pp. 1 - 36. 
SCHALLER， G.B.; CRAWSHAW, P.G. 1981. (Social organization in a capybara population. Saügetierkundl). Mitt., 29: pp. 3 - 16.

SOINI, P. 1993. Un estudio de la dinámica poblacional del ronsoco 0 capybara (Hydrochoerus hydrochaeris) en el río Pacaya, Perú. Folia A mazónica, 5: pp. 137 - 154.

SOINI, P. Y SOINI, M. 1992. Ecología del ronsoco o capybara (Hydrochoerus hydrochaeris) en la Reserva Nacional Pacaya-Samíria, Perú. Folia A mazónica, 4: pp. 119 - 133. 
Cuadro № 1. Composición de la población de ronsocos (H ydrochaeris) en el área A, en setiembre de 1979 y 1980.

\begin{tabular}{cccccccccc}
\hline $\begin{array}{c}1979 \\
\text { Unidad } \\
\text { Social } \\
\#\end{array}$ & $\begin{array}{c}\text { Adultos } \\
\text { y sub- } \\
\text { adultos }\end{array}$ & Juv. & Inf. & Tot. & $\begin{array}{c}1980 \\
\text { Unidad } \\
\text { Social } \\
\#\end{array}$ & $\begin{array}{c}\text { Adultos } \\
\text { y sub- } \\
\text { adultos }\end{array}$ & Juv. & Inf. & Tot. \\
\hline 1 & 3 & 2 & 6 & 11 & 1 & 2 & -- & 5 & 7 \\
2 & 2 & -- & -- & 2 & 2 & 2 & -- & 5 & 7 \\
3 & 2 & -- & -- & 2 & 3 & 2 & 2 & -- & 4 \\
4 & 2 & 2 & 1 & 5 & 4 & 2 & 2 & 3 & 7 \\
5 & 1 & -- & -- & 1 & 5 & 2 & -- & -- & 1 \\
6 & 1 & -- & -- & 1 & 6 & 1 & -- & -- & 1 \\
7 & 1 & -- & -- & 1 & & & & & \\
\hline
\end{tabular}

A dultos y subadultos ( $>12$ meses)

J uv. $=$ J uveniles (4-12 meses)

Inf. $=$ Infantes (0-4 meses)

Cuadro № 2. Población de ronsocos (Hydrochaeris) del área B, en setiembre-octubre de 1984

\begin{tabular}{ccccc}
\hline $\begin{array}{c}\text { Unidad } \\
\text { Social \# }\end{array}$ & $\begin{array}{c}\text { Adultos y } \\
\text { Subadultos }\end{array}$ & Juveniles & Infantes & Total \\
\hline 1 & 2 & -- & 3 & 5 \\
2 & 2 & -- & 2 & 4 \\
3 & 2 & -- & -- & 2 \\
4 & 4 & 1 & 1 & 6 \\
5 & 2 & -- & 4 & 6 \\
6 & 2 & 2 & -- & 4 \\
7 & 2 & -- & 1 & 3 \\
8 & 4 & 2 & -- & 6 \\
9 & 1 & -- & -- & 1 \\
10 & 1 & -- & -- & 1 \\
11 & 1 & -- & -- & 1 \\
\hline
\end{tabular}


C uadro № 3. Recuentos nocturnos de ronsocos en el tramo de censo del área A en setiembre-noviembre de 1979

\begin{tabular}{ccccc}
\hline Fecha & $\begin{array}{c}\text { Familias } \\
\text { (No adult. + crias) }\end{array}$ & $\begin{array}{c}\text { Parejas } \\
(\mathbf{N o} \text { indivs })\end{array}$ & Solitarios & $\begin{array}{c}\text { Total de } \\
\text { individuos }\end{array}$ \\
\hline 16.09 & $1(2+6)$ & $1(2)$ & 1 & 11 \\
17.09 & $1(5+6)$ & $1(2)$ & 3 & 16 \\
18.09 & $1(1+3)$ & -- & 3 & 7 \\
19.09 & -- & -- & 2 & 2 \\
20.09 & -- & $1(2)$ & 1 & 3 \\
23.09 & $1(1+1)$ & & -- & 2 \\
24.09 & -- & -- & 2 & 2 \\
07.10 & -- & -- & 2 & 2 \\
16.11 & $1(4+1)$ & $1(2)$ & 3 & 10 \\
17.11 & -- & -- & 1 & 1 \\
18.11 & -- & -- & 1 & 1 \\
\hline
\end{tabular}

Cuadro № 4 Relación aproximada entre la edad y la longitud total de la huella de la pisada del ronsoco (Hydrochaeris hydrochaeris) en el río Pacaya

\section{Categoría de edad}

Long. de la huella del pie trasero

Crías menores de 1 mes

Crías de 1 - 2 meses

Crías de 3 meses o más

Juveniles de 6 meses o más

Subadultos de aprox. 1 año de edad

Adultos

$4-5 \mathrm{~cm}$

$5.5-6.5 \mathrm{~cm}$

$7 \mathrm{~cm}$

$8-10 \mathrm{~cm}$

$10-11 \mathrm{~cm}$

$11-13 \mathrm{~cm}$ 
Figura 1. El A rea de estudio ----- el hábitat del ronsoco en el área A.

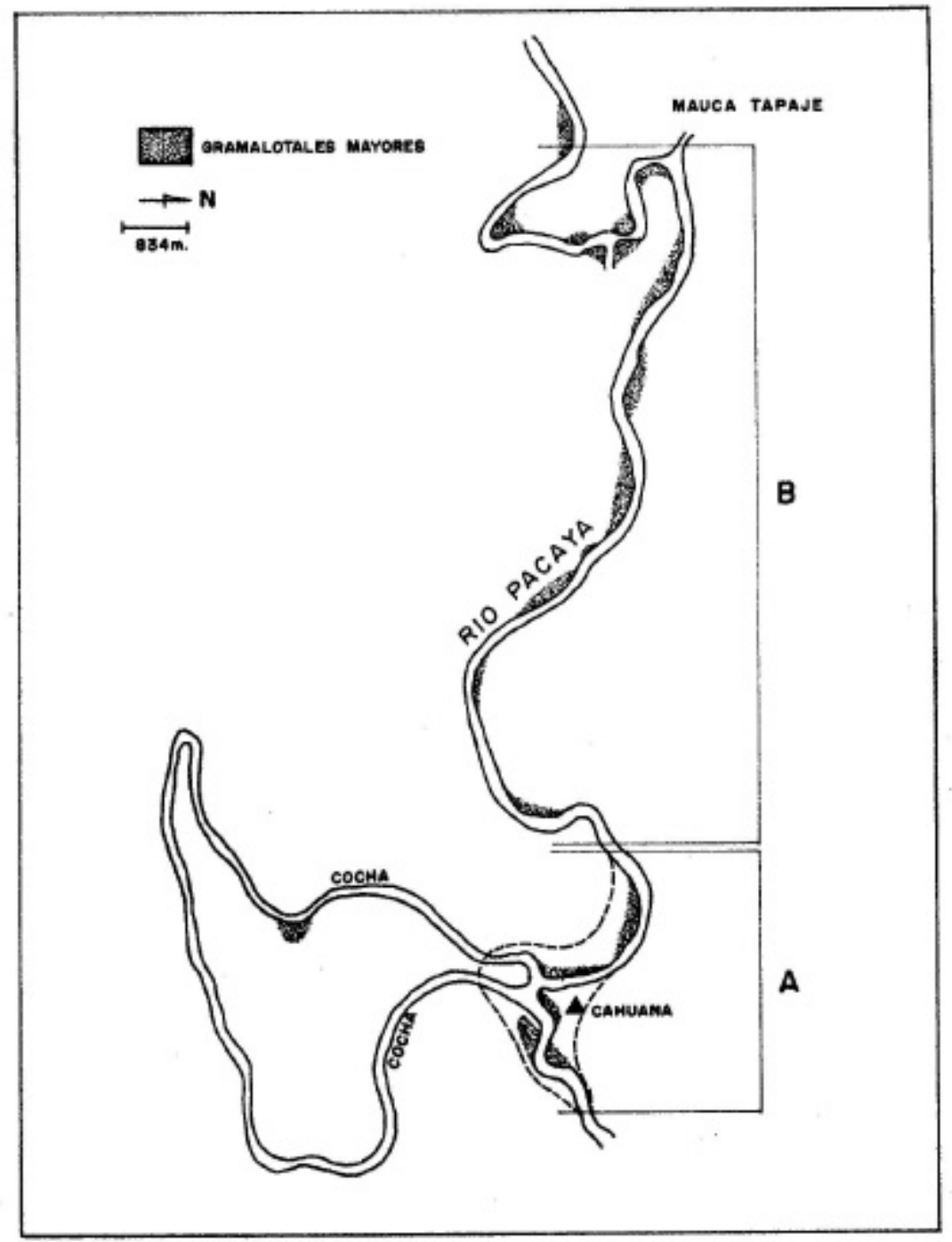

